\title{
TEMA-2017: Actualización en enfermedad ácido péptica
}
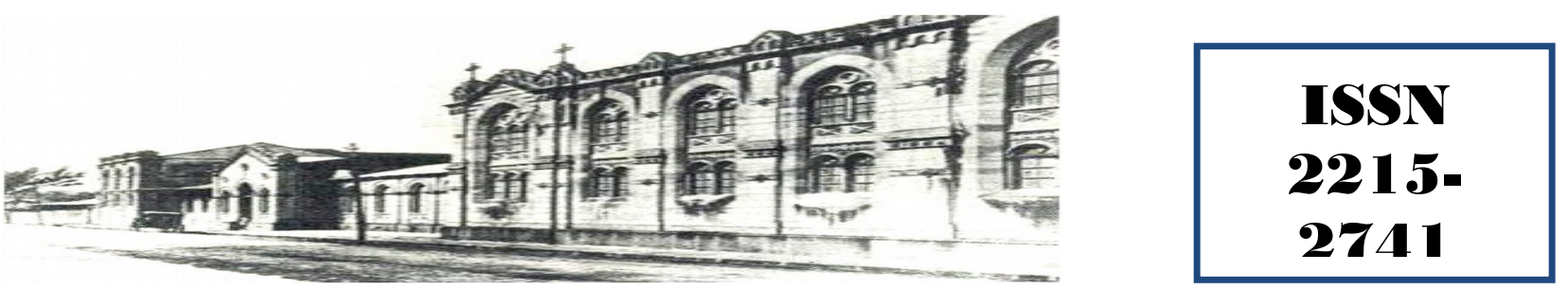

Haspital San quan de Dias. San Jasé, Casta Rica. Fundada en 1845

Recibido:

$30 / 11 / 16$

Aceptado:

$15 / 01 / 17$

Pablo Coste Murillo ${ }^{1}$

Rolando Páez Sáenz ${ }^{2}$

${ }^{1}$ Médico Especialista en Medicina Interna y residente de Gastroenterología, Hospital San Juan de Dios. Correo electrónico: costepablo@gmail.com

${ }^{2}$ Médico Especialista Gastroenterología, Hospital R. A. Calderón Guardia. Correo electrónico: rapaezsa@yahoo.com

\section{RESUMEN}

Las úlceras pépticas son defectos de la mucosa gástrica o intestinal mayores a $5 \mathrm{~mm}$ que se extienden a través de la muscular de la mucosa. Su patogénesis es multifactorial y parte del desbalance entre los factores protectores y agresores en la mucosa gastrointestinal.

A raíz de la gran cantidad de factores involucrados en la patogénesis de la EAP, hoy en día se han aceptado y clasificado de acuerdo a su principal causa etiológica: asociadas a H.pylori, daño por AINES o úlceras no asociadas a $\mathrm{H}$. pylori, ni AINES, dentro de las cuales un porcentaje cada vez menor terminan siendo idiopáticas.

La tasa de complicaciones tipo hemorragia, perforación y obstrucción confieren una mortalidad significativa, por lo cual es fundamental actualizar los principios de diagnóstico y manejo.

El manejo de acuerdo a la etiología, las indicaciones de endoscopía, la utilización de medicamentos, la aparición en presencia de antiagregantes y anticoagulación, son algunas de las muchas situaciones que generan dudas en la práctica diaria del médico.

La presente revisión propone estudiar y actualizar de una forma complementaria, los fundamentos del diagnóstico, manejo y tratamiento de la enfermedad ácido péptica desde un punto de vista práctico y esquematizado.

\section{PALABRAS CLAVE}

Enfermedad ácido péptica, enfermedad ulcerosa péptica, H pylori, AINES.

\section{ABSTRACT}

Peptic ulcers are defects of the gastric or intestinal mucosa greater than $5 \mathrm{~mm}$ that extend through the muscular mucosa. The pathogenesis is multifactorial and the result of the imbalance between protective and damaging factors in the gastrointestinal mucosa. 
Due to the large number of factors involved in the pathogenesis of PUD, now a day they areclassified according to their etiology: H.pylori-associated, NSAID or Non H Pylori non NSAID ulcers, of which a decreasing percentage end up being idiopathic.

The complications rate, including hemorrhage, perforation and obstruction confer significant mortality, which is why it is fundamental to update the principles of its diagnosis and management.

Management according to the etiology, endoscopy indications, the use of medications, the incidence while using antiplatelet and anticoagulants drugs, are some of the many situations that generate doubts in the physicians daily practice.

This revision pretends to update the diagnosis, management and treatment of peptic acid disease from a practical point of view, in a complementary way.

\section{KEY WORDS}

Peptic acid disease, ulcerous disease, $\mathrm{H}$ pylori, NSAID's.

\section{INTRODUCCIÓN}

La medicina constituye una ciencia cada vez más especializada, con una tendencia constante hacia la especificación de la patología y su manejo.

Dentro de esta dinámica, es imprescindible para el médico lograr actualizar sus conocimientos en cuanto a los fundamentos de las enfermedades de aparición frecuente y que afectan a los pacientes independientemente de sus otras comorbilidades.

La enfermedad ácido péptica toma un papel importante es este sentido. Su alta incidencia y prevalencia, aunada a una morbimortalidad significativa, la convierten en una patología de manejo diario independientemente de la especialidad involucrada.

Esta revisión se fundamenta en esta necesidad e intenta repasar los principios básicos de esta enfermedad con el fin de orientar el estudio complementario de la misma.

\section{DISCUSIÓN}

\section{Generalidades}

Las úlceras pépticas son defectos de la mucosa gástrica o intestinal mayores a $5 \mathrm{~mm}$ que se extienden a través de la muscular de la mucosa. Su patogénesis es multifactorial y parte del desbalance entre los factores protectores y agresores en la mucosa gastrointestinal.

Previamente se consideraba una enfermedad idiopática y crónica sin embargo esta situación cambió en 1984 con la identificación de Helicobacter pylori como un agente nocivo y tratable. Con la identificación de los AINES y la aspirina como factores de riesgo mayor se evidenció que sólo una pequeña fracción de las úlceras se encuentra asociada a trastornos neoplásicos, estados de hipersecreción ácida, fumado, otros medicamentos, enfermedades poco frecuentes y trastornos idiopáticos. ${ }^{(1)}$

Desde 1950, la incidencia y prevalencia de las úlceras gástricas (UG) y duodenales (UD) ha disminuido constantemente en respuesta a los avances en cuanto a tratamiento y menor prevalencia de H. pylori en relación a mejores condiciones sanitarias y acceso a antibióticos.

Esto explica por qué en los Estados Unidos, la tasa de hospitalización ajustada por edad para enfermedad úlcero péptica (EUP) y H. pylori fue más elevada en los pacientes mayores de 65 años y decreció con cada grupo etario subsecuente. ${ }^{(2,3)}$

Además, el impacto económico y social de esta enfermedad es sustancial, estimando los gastos directos e indirectos en 10 billones de dólares anuales en los Estados Unidos. ${ }^{(4)}$

De estos factores deriva la importancia de conocer el diagnóstico, manejo y tratamiento de esta patología.

\section{Epidemiología}

La tendencia mundial es a la disminución progresiva y constante de la incidencia y complicaciones de la EUP, principalmente en los pacientes menores de 60 años. Esto se debe a la interacción de múltiples factores: mejoría de las condiciones de salud y socioeconómicas 
posterior a la Segunda Guerra Mundial, mayor uso de AINES en el adulto mayor, disminución en el fumado, etc. ${ }^{(5)}$

Una revisión sistemática de EUP estimó una incidencia anual de úlcera diagnosticada por el médico de 0.1 a $0.19 \%$ y de 0.03 a $0.17 \%$ diagnosticadas durante la hospitalización3. La incidencia agrupada y la tasa de sangrado (0.019 vs 0.024) para la UG y UD son comparables mientras que la de perforación es mayor en la UD (0.0055 vs 0.0014). ${ }^{(6)}$ Ambos tipos de úlceras aumentan su incidencia y riesgo de complicaciones con la edad siendo el riesgo de sangrado 13 veces mayor en pacientes $>70$ años que en $<40$ años. ${ }^{(6)}$

En cuanto a la prevalencia, se estima que es de 0.12 a $1.5 \%$ por año basadas en el diagnóstico del médico y de 0.1 a $0.19 \%$ basado en hospitalizados, siendo la prevalencia de por vida de aproximadamente $12 \%$ en hombres y $10 \%$ en mujeres. ${ }^{(1,3)}$

\section{Patogénesis}

Las úlceras son la última consecuencia de un desequilibrio entre factores agresores $y$ protectores. Los principales agresores son el ácido estomacal y las secreciones gástricas con alta actividad proteolítica (pepsina) mientras que los principales protectores se enumeran en la Tabla 1. ${ }^{(7)}$

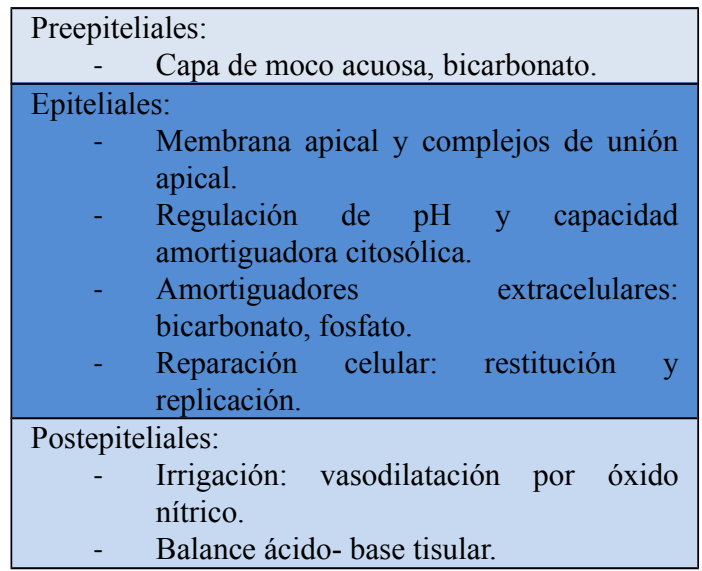

Tabla 1: Factores protectores de la mucosa gástrica Fuente: Basado en Tytgat, 2011.
En resumen, los factores etiológicos alteran la capacidad normal de reparación y defensa de la mucosa haciéndola más susceptible al ácido. ${ }^{(1)}$ Distintos factores pueden representar importantes noxas y favorecer el daño del epitelio. (Ver Tabla 2)

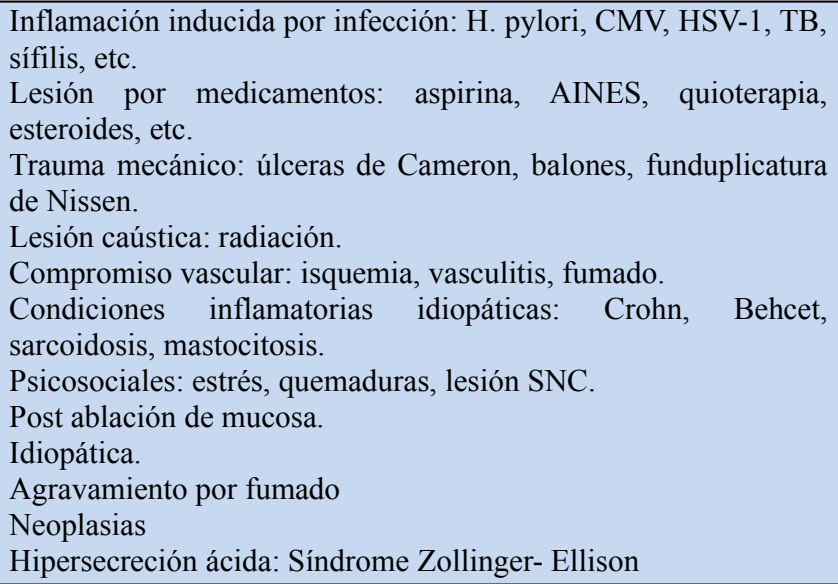

Tabla 2: Causas multifactoriales de úlceras

Fuente: Basado en Tytgat, 2011.

A raíz de la gran cantidad de factores involucrados en la patogénesis de la EAP, hoy en día se han aceptado y clasificado de acuerdo a su principal causa etiológica en asociadas a H.pylori, daño por AINES o idiopáticas (No H. pylori, no AINES). ${ }^{(7)}$

\section{H. pylori}

Se trata de una bacteria gram negativa productora de ureasa que se encuentra en la mucosa gástrica y ha sido clasificado como carcinógena y una causa definitiva de cáncer gástrico en el ser humano. ${ }^{(1)}$

Múltiples factores como la ureasa le permiten alcalinizar el $\mathrm{pH}$ gástrico y sobrevivir en este ambiente causando lesiones en la mucosa y úlceras mediante la cascada inflamatoria y citokinas. La presencia de factores de virulencia del organismo (VacA, BabA, CagA, etc), la genética del huésped y el ambiente afectan la distribución y severidad de la inflamación gástrica. ${ }^{(1)}$

La incidencia de úlceras en pacientes portadores es de un $1 \%$, de 6 a 10 veces más riesgo que en 
no portadores y la prevalencia de por vida es de aproximadamente el doble (10-20\%) en pacientes con la bacteria comparado con la población general8.

En los países en desarrollo, la mayoría de los niños son infectados por $\mathrm{H}$. pylori antes de los 10 años y la prevalencia es mayor al $80 \%$ para la quinta década. En los países desarrollados sólo hay evidencia serológica en el $50 \%$ de los pacientes mayores de 60 años. ${ }^{(9)}$

En nuestro país, en estudios realizados con pacientes del Hospital México durante la década de los 90, se evidenció que la prevalencia de aislamiento de la bacteria en los casos de gastritis osciló alrededor del $70 \%$, en tanto que en úlceras pépticas fue del 80 al 90\%. En el 2001 se publicó un estudió que reportó una prevalencia del $54 \%$ y un perfil típico de infección temprana en la vida con cronicidad, dado que su prevalencia aumentó con la edad. ${ }^{(10,11,12)}$

\section{AINES/ aspirina}

Más de 30 millones de personas utilizan estos medicamentos diariamente y hasta $30-50 \%$ presentan lesiones endoscópicas tipo petequias, erosiones y úlceras. Estudios epidemiológicos sugieren que el riesgo de úlceras y muerte en los usuarios es de 3-10 veces más elevado, siendo los adultos mayores los más susceptibles con una tasa de exceso de hospitalizaciones por EUP de 12.5 por 1000 personas- año dentro de los usuarios. ${ }^{(13)}$

Estos fármacos ejercen su efecto tóxico mediante la inhibición de la COX-1, la enzima limitante en la producción de prostaglandinas que normalmente ayuda a mantener la irrigación de la mucosa y aumenta la secreción de moco y bicarbonato. $^{(1)}$

La toxicidad gastrointestinal depende de las características clínicas del paciente, asi como de la clase química, dosis, formulación y vida media del fármaco. ${ }^{(13)}$ Aún pequeñas dosis de aspirina se han asociado a EUP. (Ver tabla 3 y 4 ).

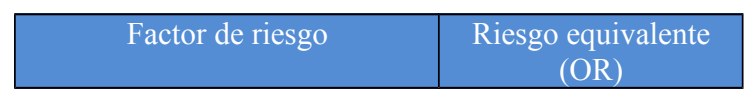

\begin{tabular}{|c|c|}
\hline Úlcera complicada previa & 13.5 \\
AINES+ anticoagulante & 12.7 \\
Múltiples AINES & 9 \\
AINES a altas dosis & 7 \\
AINES con ISRS & 6.33 \\
Úlcera previa no complicada & 6.1 \\
AINES+ dosis baja de aspirina & 5.6 \\
$>65$ años & 3.5 \\
H. pylori & 3.5 \\
2 o más comorbilidades & 2.26 \\
\hline
\end{tabular}

Tabla 3: Factores de riesgo para EUP asociada a AINES.

Fuente: Basado en Melcarne et al, 2016.

\begin{tabular}{|l|l|}
\hline \multicolumn{1}{|c|}{ Medicamento } & \multicolumn{1}{c|}{ RR $(95 \% \mathrm{IC})$} \\
\hline Naproxeno & $4.10(3.22-5.23)$ \\
Ibuprofeno & $1.84(1.54-2.00)$ \\
Diclofenaco & $3.34(2.79-3.99)$ \\
Indometacina & $4.14(2.91-5.90)$ \\
Celecoxib & $1.45(1.17-1.81)$ \\
Rofecoxib & $2.32(1.89-2.86)$ \\
\hline
\end{tabular}

Tabla 4: Riesgo relativo acumulado de AINES para eventos gastrointestinales.

Fuente: Basado en Melcarne et al, 2016.

\section{Idiopática}

Se define como una úlcera de causa desconocida o que impresiona aparecer espontáneamente, de manera que cuando todas las causas conocidas son descartadas se puede establecer el diagnóstico de esta entidad. En diversos estudios se ha evidenciado que la proporción de úlceras no asociadas a $\mathrm{H}$ pylori ni AINES varía entre el 20 al 44\%, siendo el porcentaje de úlceras idiopáticas una fracción cada vez menor. ${ }^{(14)}$

Comparativamente con las úlceras duodenales por $\mathrm{H}$ pylori, las idiopáticas tienden a asociarse a mayor edad, mayor comorbilidad, antecedente de neoplasia, cirugía reciente, sepsis, isquemia mesentérica, fumado, estrés e hipersecreción ácida. ${ }^{(7,15)}$

Clínicamente se asocian a mayor riesgo de complicaciones, peor respuesta a la inhibición ácida y mayor tasa de recurrencia. ${ }^{(15)}$ Los pacientes que presentan sangrado por estas úlceras presentan un aumento de cuatro veces del riesgo de sangrado recurrente y mortalidad. ${ }^{(1)}$ 
Al ser un diagnóstico de exclusión, debe hacerse un esfuerzo terapéutico por descartar etiologías posibles: falsos negativos de $\mathrm{H}$ pylori, uso subrepticio de medicamentos ulcerogénicas y las entidades descritas en la Tabla 2.

\section{Clínica}

El síntoma clásico es la dispepsia, descrita subjetivamente como un dolor epigástrico o incomodidad urente, sordo, "vacío" o "como sensación de hambre". Generalmente, el dolor asociado a la UD tiende a aliviarse con la ingesta de alimentos pero recurre a las 2-4 horas y despierta al paciente en la noche mientras que el de la UG empeora con la ingesta y por ende puede asociarse a nausea e hiporexial. Los pacientes con UD tienden a ser más jóvenes, frecuentemente entre los 30 y 55 años, mientras que las UG ocurren entre los 55 y 70 años, con una incidencia mayor en la sexta década.

Aproximadamente $70 \%$ de las úlceras pépticas son asintomáticas siendo los adultos mayores y los individuos con uso de AINES los más frecuentes. ${ }^{(16)}$

La clínica tiende a ser recurrente y cambios en el patrón usual o la presencia signos de alarma pueden deberse a complicaciones de la EUP. Por ejemplo, la presencia de melena, hematemesis, guayacos positivos o anemia puede sugerir sangrado, los vómitos persistentes obstrucción, el dolor con irradiación dorsal penetración y el dolor súbito perforación. ${ }^{(1)}$

$\mathrm{Al}$ examen físico los hallazgos son inespecíficos $\mathrm{y}$ frecuentemente ausentes. Datos indirectos como taquicardia, ortostatismo, rigidez abdominal, rebote, distensión abdominal y otros pueden generar la sospecha de EUP complicada. (1)

\section{Diagnóstico}

\section{Endoscópico}

El diagnóstico definitivo de la EUP se establece mediante la visualización directa de la úlcera mediante el estudio endoscópico. La sensibilidad para la detección de lesiones gastroduodenales es de aproximadamente $90 \% .{ }^{(17)}$
Todas las úlceras con aspecto maligno deben ser biopsiadas a diferencia de las UD de aspecto benigno pues raramente malignizan. En nuestro país y debido a la alta incidencia de cáncer gástrico, es razonable tomar biopsia de toda UG y repetir la endoscopía a las 12 semanas post tratamiento con el fin de confirmar curación completa, ya que hasta el $5 \%$ son malignas. ${ }^{(1)}$

Algunos de los hallazgos endoscópicos que sugieren malignidad son: masa ulcerada protruyente, pliegues nodulares alrededor del cráter ulceroso, fusionados o que terminan antes del margen ulceroso, bordes irregulares y engrosados, localizaciones atípicas, etc. ${ }^{(1,17)}$

Pacientes sospechosos de ser portadores de EUP que se presentan con signos de alarma como sangrado gastrointestinal, saciedad temprana, pérdida de peso o adulto mayor deben someterse al estudio endoscópico. ${ }^{(1)}$

En contraste, hay controversia en cuanto al manejo del paciente menor de 55 años sin signos de alarma que se presentan con sintomatología de EAP o dispepsia. Dentro de las posibilidades de manejo que se encuentran: 1) testear y tratar por H. Pylori, 2) tratar con supresores de ácido y monitorizar respuesta o 3) realizar endoscopía. Ninguno de los abordajes ha demostrado superioridad por sobre los otros, pero los tres recomiendan discontinuar la aspirina/ AINES, alcohol, fumado y las drogas ilícitas. ${ }^{(18)}$

Imagen

La utilización de estudios con bario es infrecuente debido a su baja sensibilidad para detectar úlceras o lesiones de la mucosa, así como la incapacidad de permitir el estudio histológico o manejo terapéutico. ${ }^{(1)}$

\section{Estudio etiológico}

\begin{tabular}{|l|l|l|l|}
\hline \multicolumn{1}{|c|}{ Test } & $\begin{array}{c}\text { Sensi- } \\
\text { bili- } \\
\text { dad } \\
(\%)\end{array}$ & $\begin{array}{c}\text { Espe- } \\
\text { cifici- } \\
\text { dad } \\
(\%)\end{array}$ \\
\hline & & 79 & $\begin{array}{l}\text { No funciona para } \\
\text { confirmar erradi- } \\
\text { cación }\end{array}$ \\
\hline $\begin{array}{l}\text { ELISA } \\
\text { serología }\end{array}$ & 85 & $91-98$ & $\begin{array}{l}\text { Para tamizaje y } \\
\text { confirmación. }\end{array}$ \\
\hline $\begin{array}{l}\text { Urea en } \\
\text { aliento }\end{array}$ & $95-100$ & & \\
\hline
\end{tabular}




\begin{tabular}{|c|c|c|c|}
\hline & & & $\begin{array}{l}\text { Falsos negativos: } \\
\text { uso reciente ATB } \\
\text { e IBP. }\end{array}$ \\
\hline $\begin{array}{l}\text { Antígeno } \\
\text { en heces }\end{array}$ & $91-98$ & $94-99$ & $\begin{array}{l}\text { Para diagnóstico } \\
\text { inicial y confir- } \\
\text { mación. }\end{array}$ \\
\hline \multicolumn{4}{|l|}{ Invasivo } \\
\hline $\begin{array}{l}\text { Histolo- } \\
\text { gía }\end{array}$ & $>95$ & $95-98$ & $\begin{array}{l}\text { Alto valor econó- } \\
\text { mico. }\end{array}$ \\
\hline $\begin{array}{l}\text { Test de } \\
\text { ureasa } \\
\text { rápido }\end{array}$ & $93-97$ & $\begin{array}{l}95- \\
100\end{array}$ & $\begin{array}{l}\text { Menor precisión } \\
\text { en sangrado GI. }\end{array}$ \\
\hline Cultivo & $70-80$ & 100 & $\begin{array}{l}\text { Técnicamente } \\
\text { muy demandante. }\end{array}$ \\
\hline
\end{tabular}

Tabla 4: Test diagnóstico para $\mathrm{H}$ pylori.

Fuente: Basado en Lew et al, 2016.

\section{Diagnóstico diferencial}

Reflujo gastroesofágico, enfermedades del árbol biliar, hepatitis, pancreatitis, aneurisma aorta abdominal, gastroparesia, dispepsia funcional, neoplasia, isquemia mesentérica, isquemia miocárdico, entre otros.

\section{Complicaciones}

En los Estados Unidos la hemorragia es la complicación más frecuente $(73 \%)$, seguido por la perforación $(9 \%)$ y la obstrucción $(3 \%)$. En cuanto a mortalidad es mayor la perforación, seguido por la obstrucción y la hemorragia. ${ }^{(19)}$

Hasta el $15 \%$ de las UP sangran, teniendo una mortalidad del 4.5-8.5\%. Ambos porcentajes aumentan en el adulto mayor y en pacientes que se presentan con sangrados profusos, continuos o recurrentes, con mucha comorbilidad, poca reserva fisiológica o úlceras mayores a $2 \mathrm{~cm}$. En perforación, datos de peritonitis, un retraso de la cirugía mayor a 12 horas o UG por sobre UD confieren peor pronóstico, al igual que un pobre estado nutricional en los casos de obstrucción. La penetración ocurre cuando el cráter ulceroso erosiona hacia órganos adyacentes. ${ }^{(1,20)}$

\section{Tratamiento}

\section{General}

En cuanto al abordaje inicial en pacientes con úlceras complicadas, deben instaurarse las siguientes medidas: iniciar monitoreo, reanimación con fluidoterapia y hemocomponentes de con la meta de $\mathrm{Hb}>7 \mathrm{~g} / \mathrm{dL}$, comenzar dosis intravenosa plena de Inhibidores de Bomba de Protones (80mg IV STAT seguido por $8 \mathrm{mg} / \mathrm{h}$ y continuar por $72 \mathrm{hrs}$ ) y realizar endoscopía para posible resolución terapéutica en las primeras 24 horas en la mayoría de los casos o en cuanto se estabilice el paciente en el contexto de una hemorragia severa. ${ }^{(1,20,21)}$

Una vez que el paciente tolera la vía oral, debe iniciarse IBP VO BID, tratar por H. Pylori si es positivo, eliminar los AINES en la medida de lo posible, detener la aspirina si el status cardiovascular lo permite, repetir la endoscopía a las 8-12 semanas en caso de UG para excluir neoplasia o UD gigantes y cambiar a dosis plenas de antagonistas de receptor $\mathrm{H} 2$ a las 4 semanas si los estudios por H. pylori son negativos. ${ }^{(1,20,21)}$

\section{Úlceras asociadas a H. pylori}

Hay varias líneas de tratamiento (Ver Tabla 5). Se recomienda confirmar la erradicación 4 a 8 semanas posterior al término del tratamiento. ${ }^{(23)}$

\begin{tabular}{|c|c|c|}
\hline Tratamiento & $\begin{array}{l}\text { Erradica- } \\
\text { ción }\end{array}$ & Comentarios \\
\hline $\begin{array}{l}\text { Todos BID: IBP+ } \\
\text { claritromicina } \\
500 \mathrm{mg} \text { ó metroni- } \\
\text { dazol 500mg+ } \\
\text { amoxicilina } 1 \mathrm{~g} \text {, } \\
\text { por } 10-14 \mathrm{~d} \text {. } \\
\text { Todos BID: IBP+ } \\
\text { claritromicina } \\
500 \mathrm{mg}+\text { metroni- } \\
\text { dazol 500mg, por } \\
10-14 \mathrm{~d} \text {. }\end{array}$ & $64-85 \%$ & $\begin{array}{l}\text { No si la resis- } \\
\text { tencia a clari- } \\
\text { tromicina es } \\
>15 \% \text { en la } \\
\text { población. } \\
\text { Puede usarse } \\
\text { como primera } \\
\text { línea en alér- } \\
\text { gicos a la pe- } \\
\text { nicilina. }\end{array}$ \\
\hline $\begin{array}{l}\text { Cuádruple: subs- } \\
\text { alicilato bismuto } \\
525 \mathrm{mg} \text { QID+ me- } \\
\text { tronidazol } 250 \mathrm{mg} \\
\text { QID+ Tetraciclina } \\
50 \mathrm{mmg} \text { QID+ IBP } \\
\text { BID, por } 10-14 \mathrm{~d} \text {. }\end{array}$ & $75-90 \%$ & $\begin{array}{l}\text { Puede ser de } \\
\text { primera línea } \\
\text { en zonas con } \\
\text { alta resisten- } \\
\text { cia a claritro- } \\
\text { micina. }\end{array}$ \\
\hline $\begin{array}{l}\text { Secuencial con } \\
\text { claritromicina: } \\
\text { IBP BID+ amoxi- } \\
\text { cilina } 1 \mathrm{~g} \text { BID por } \\
5 \mathrm{~d}+\text { claritromicina } \\
500 \mathrm{mg} \text { BID y tini- }\end{array}$ & $60-83 \%$ & $\begin{array}{l}\text { Puede ser de } \\
\text { primera línea. }\end{array}$ \\
\hline
\end{tabular}




\begin{tabular}{|c|c|}
\hline \multicolumn{2}{|l|}{$\begin{array}{l}\text { dazol 500mg BID } \\
\text { los siguientes } 5 \mathrm{~d} \text {. }\end{array}$} \\
\hline $\begin{array}{l}\text { Triple con levoflo- } \\
\text { xacina: IBP BID+ } \\
\text { levofloxacina } 250- \\
500 \mathrm{mg} \text { BID+ } \\
\text { amoxicilina } 1 \mathrm{~g} \\
\text { BID, por } 10-14 \mathrm{~d} \text {. }\end{array}$ & \\
\hline $\begin{array}{l}\text { Otras: secuencial } \\
\text { con levofloxacina, } \\
\text { cuádruple sin bis- } \\
\text { muto, híbrida (Ver } \\
\text { Lew et al.) }\end{array}$ & \\
\hline & \\
\hline $\begin{array}{l}\text { No recomendada } \\
\text { empíricamente. }\end{array}$ & $\begin{array}{l}\text { Debe guiarse } \\
\text { de acuerdo a } \\
\text { la sensibili- } \\
\text { dad local. }\end{array}$ \\
\hline
\end{tabular}

Tabla 5: Tratamientos para H. pylori.

Fuente: Basado en Lew et al, 2016.

En las UD no complicadas asintomáticas, no es necesario la utilización de terapia antisecretora posterior al tratamiento de erradicación. En los casos de UD complicadas o UG, se sugiere IBP por 4-8 semanas y 8-12 respectivamente. En las UG debe discontinuarse sólo hasta corroborar curación completa endoscópicamente. ${ }^{(23)}$

\section{Úlceras asociadas a AINES/ aspirina}

Los siguientes son principios aceptados del manejo de los pacientes con EUP en el contexto de uso de AINES/ aspirina ${ }^{(13)}$ :

- Los AINES/ aspirina deben discontinuarse en la medida de lo posible, y utilizar opciones alternativas tipo acetaminofén.

- En presencia de EUP, los pacientes deben recibir IBP por un mínimo de 8 semanas. En caso de ocupar AINES permanentemente, deben utilizarse a la menor dosis posible y acompañados de IBP o misoprostol. Ambos tienen similar capacidad de prevención de úlceras pero los IBP son mejor tolerados.
- Los inhibidores COX-2 (Coxib) tienen similar efecto analgésico y antiinflamatorio que los AINES pero con menos riesgo de efectos gastrointestinales adversos.

- Los IBP disminuyen el riesgo de EUP asociada a AINES y son superiores a los Bloqueadores de H2 en la prevención de lesiones gástricas.

- Idealmente debe procurarse erradicación de H. pylori antes del inicio de AINES.

- La elección de un analgésico debe valorarse con respecto al riesgo cardiovascular y gastrointestinal individual. Ver Tabla 6.

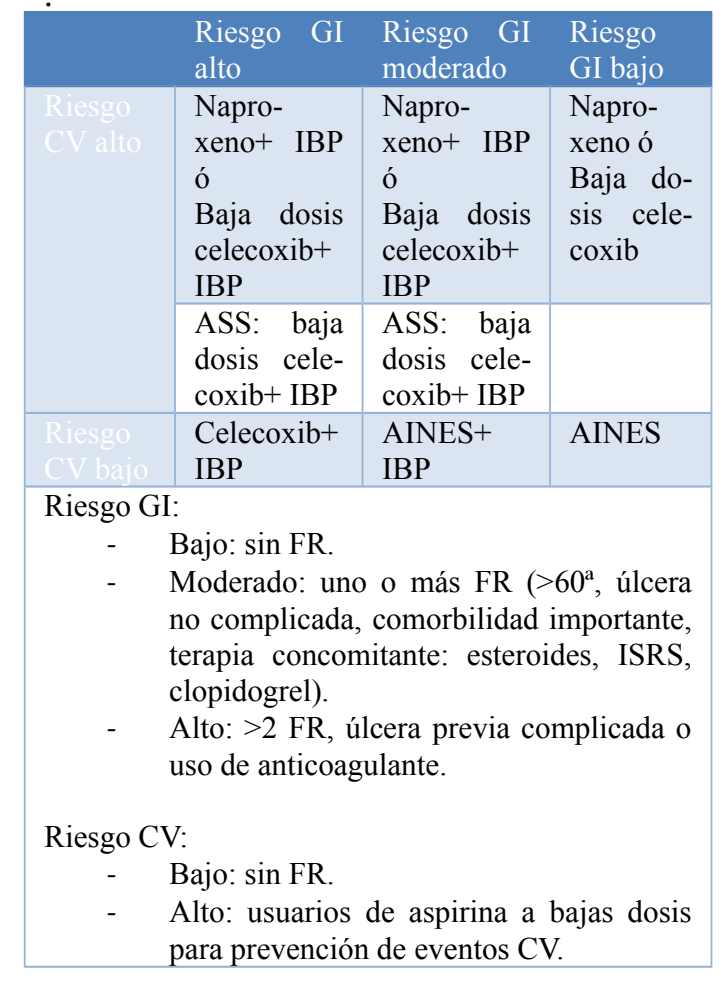

Tabla 6: Uso de AINES de acuerdo al riesgo cardiovascular y gastrointestinal 
Fuente: Basado en Melcarne et al, 2016.

Úlceras en el contexto de antiagregantes/ anticoagulantes

Los siguientes son principios aceptados del manejo de los pacientes con EUP en el contexto de uso antiagregantes/ anticoagulantes. ${ }^{(13)}$

- La aspirina representa una importante noxa gastrointestinal aún a dosis bajas pero debe reintroducirse al tercer día posterior al sangrado cuando sea la antiagregación sea necesaria.

- Clopidogrel tiene mayor riesgo de sangrado que la aspirina+ IBP por lo que debe cambiarse a esta combinación cuando sea posible.

- En doble antiagregación, debe mantenerse la aspirina y detener el clopidogrel momentáneamente.

- En los pacientes con sangrados que no amenacen la vida (Forrest IIc y III) la opción con menor riesgo es mantener la anticoagulación.

- En los que amenazan la vida, debe revertirse la anticoagulación e iniciar la HBPM en cuanto el sangrado se controle. Debe reiniciarse la antIcoagulación oral a los 7-15 días.

Úlceras No asociadas a H.pylori ni AINES e idiopáticas

De acuerdo a las guías japonesas para el manejo de la EAP, los IBP son el tratamiento de elección en estos casos. ${ }^{(23)}$ Se sugiere su uso durante 4 a 8 semanas y descartar la presencia de H. pylori o uso subrepticio de AINES. En su ausencia se recomienda la utilización continua. ${ }^{(22)}$

\section{Seguimiento}

La endoscopía control se recomienda al término de 12 semanas de IBP en el caso de UD con síntomas persistentes o UG con cualquiera de las siguientes condiciones: síntomas persistentes, etiología incierta, úlcera gigante $(>2 \mathrm{~cm})$, biopsias no tomadas en la endoscopía inicial, úlceras de aspecto maligno, endoscopía inicial por sangrado, factores de riesgo para cáncer gástrico $\left(>50^{\mathrm{a}}, \mathrm{H}\right.$. pylori, residentes en zonas de alta prevalencia, AHF de $\mathrm{Ca}$ gástrico, atrofia gástrica, adneoma, displasia, metaplasia intestinal). ${ }^{(22)}$

Se recomienda el mantenimiento del IBP en los siguientes casos de EUP: úlcera gigante $(>2 \mathrm{~cm})$ y $>50^{\mathrm{a}}$ ó múltiples comorbilidades, úlceras idiopáticas, úlcera péptica refractaria (úlcera que no cura después de 12 semanas de IBP), fallo terapéutico en $H$. pylori, úlcera recurrente $(>2$ recurrencias en un año), uso continuo de AINES22.

\section{CONCLUSIONES}

La enfermedad ácido péptica constituye un tema amplio y frecuente an el quehacer del médico. Esta revisión intenta repasar los principios básicos de esta enfermedad y plantea 10 perlas de manejo con el fin de orientar el estudio complementario de la misma.

1. Las úlceras son consecuencia de un desequilibrio entre factores agresores y protectores gástricos y se clasifican etiológicamente en: asociadas a H.pylori, daño por AINES y úlceras No asociadas a $\mathrm{H}$. pylori, ni AINES). Dentro de este último grupo, las idiopáticas son un diagnóstico de exclusión que amerita descartar falsos negativos de $\mathrm{H}$ pylori y uso subrepticio de medicamentos ulcerogénicos.

2. La EUP tiende a ser asintomática, por lo que debe sospecharse en presencia de factores de riesgo como antecedente de úlcera, uso de AINES y/ó anticoagulantes y adultos mayores.

3. La endoscopía debe recomendarse ante sospecha de EUP en presencia de signos de alarma ó en >55 años.

4. $5 \%$ de las UG son malignas, por lo que es recomendable tomar biopsia y repetir la endoscopía a las 12 semanas post tratamiento para confirmar curación.

5. La hemorragia es la complicación más frecuente hasta en el 15\%, seguido por la perforación y la obstrucción. 
6. En las UD no complicadas asintomáticas, no es necesario la utilización de terapia antisecretora posterior al tratamiento de erradicación, mientras que en las UD complicadas o UG, se sugiere IBP por 4-8 semanas y 8-12 respectivamente.

7. En la EUP en el contexto de uso de AINES/ aspirina, la elección de un analgésico debe valorarse con respecto al riesgo cardiovascular y gastrointestinal individual.

8. En la EUP en el contexto de antiagregantes/ anticoagulantes, ante un sangrado en doble antiagregación, debe mantenerse la aspirina. En anticoagulación con sangrados que amenazan la vida, debe revertirse la anticoagulación, iniciar la HBPM en cuanto el sangrado se controle y reiniciar la anticoagulación oral a los 715 días.

9. La endoscopía control se recomienda al término de 12 semanas de IBP en el caso de UD con síntomas persistentes o UG con cualquiera de las siguientes condiciones: síntomas persistentes, etiología incierta, úlcera gigante $(>2 \mathrm{~cm})$, biopsias no tomadas en la endoscopía inicial, úlceras de aspecto maligno, endoscopía inicial por sangrado, factores de riesgo para cáncer gástrico $\left(>50^{\mathrm{a}}, \mathrm{H}\right.$. pylori, residentes en zonas de alta prevalencia, AHF de Ca gástrico, atrofia gástrica, adneoma, displasia, metaplasia intestinal).

10. Se recomienda el mantenimiento del IBP en los siguientes casos de EUP: úlcera gigante $(>2 \mathrm{~cm})$ y $>50^{\mathrm{a}} \quad$ ó múltiples comorbilidades, úlceras idiopáticas, úlcera péptica refractaria (úlcera que no cura después de 12 semanas de IBP), fallo terapéutico en $\mathrm{H}$. pylori, úlcera recurrente (>2 recurrencias en un año), uso continuo de AINES.

\section{BIBLIOGRAFÍA}

1. Lew E. Peptic Ulcer Disease. Current Diagnosis and Treatment 3rd Edition. 2016; III: 197-208.
2. Sandler RS, Everhart JE, Donowitz M, et al. The burden of selected digestive diseases in the United States. Gastroenterology. 2002; 1500- 1511.

3. Sung JJ, Kuipers EJ, El- Serag HB. Systematic review: the global incidence and prevalence of peptic ulcer disease. Aliment Pharmacol Ther. 2009; 29: 938- 946.

4. Feinstein LB, Holman RC, Yorita KL, et al. Trends in hospitalizations for peptic ulcer disease, United States, 1998- 2005. Emerg Infect Dis. 2010; 16: 1410- 1418.

5. Sonnenberg A. Temporal trends and geographical variations of peptic ulcer disease. Aliment Pharmacol Ther 1995; 9 Suppl 2:3.

6. Lin KJ, García LA, Hernández S. Systematic review of peptic ulcer disease incidence rates: do studies without validation provide reliable estimates? Pharmacoepidemiol Drug Saf 2011; 20:718.

7. Tytgat G. Etiopathogenetic Principles and Peptic Ulcer Disease Classification. Dig Dis 2011; 29:454-458.

8. Kuipers EJ, Thijs JC, Festen HP. The prevalence of Helicobacter pylori in peptic ulcer disease. Aliment Pharmacol Ther 1995; 9 Suppl 2:59.

9. Pounder RE, Ng D. The prevalence of Helicobacter pylori infection in different countries. Aliment Pharmacol Ther 1995; 9 Suppl 2:33.

10. Sigarán $M$, Hernández $F$, Rivera $P$, et al. Incidencia del Helicobacter pylori en la consulta endoscópica de un hospital costarricense. Patología 1992; 30:15-9.

11. Hernández F, Rivera P, Sigarán $M$, et al. The first cases of Campylobacter (Helicobacter) pylori reported from Costa Rica. Rev Biol Trop 1990; 38: 481-2.

12. Blanco $C$, Rivera $P$, Hernández $F$, et al. Prevalencia de Helicobacter pylori en pacientes atendidos en el Hospital San Juan 
de Dios, San José, Costa Rica. Rev Biomed 2001; 12:1-4.

13. Melcarne L., Garcia $P$, Calvet $X$. Management of NSAID-associated peptic ulcer disease. Expert Review of Gastroenterology \& Hepatology. 2016.

14. Charpignon C, Lesgourges B, Pariente A, et al. Peptic ulcer disease: one in five is related to neither Helicobacter pylori nor aspirin/NSAID intake. Aliment Pharmacol Ther. 2013; 38: 946-954.

15. Chen C, Tsung C, Yi L. A systematic approach for the diagnosis and treatment of idiopathic peptic ulcers. Korean J Intern Med 2015;30:559-570.

16. Lu CL, Chang SS, Wang SS, et al. Silent peptic ulcer disease: frequency, factors leading to "silence," and implications regarding the pathogenesis of visceral symptoms. Gastrointest Endosc 2004; 60:34.

17. Vakil N, Feldman M, Grover S. Peptic ulcer disease: Clinical manifestations and diagnosis. UpToDate 2016.

18. Delaney BC, Qume M, Moayyedi $P$, et al. Helicobacter pylori test and treat versus proton pump inhibitor in initial management of dyspepsia in primary care: multicenter randomized controlled trial. BMJ. 2008; 336: 651- 654 .

19. Wang YR, Richter JE, Dempsey DT. Trends and outcomes of hospitalizations for peptic ulcer disease in the United States, 1993 to 2006. Ann Surg 2010; 251:51.

20. Vakil N. Overview of the complications of peptic ulcer disease. UpToDate 2016.

21. Barkun AN, Bardou M, Kuipers EJ, et al. International consensus recommendations on the management of patients with nonvariceal upper gastrointestinal bleeding. Ann Intern Med. 2010; 152: 101- 113.

22. Vakil N. Peptic ulcer disease: Management. UpToDate 2016.
23. Kiichi S, Junji Y., Taiji A., et al. Evidencebased clinical practice guidelines for peptic ulcer disease 2015. J Gastroenterol (2016) 51:177-194.

\section{CONFLICTO DE INTERÉS Y/O AGRADECIMIENTOS}

Los autores declaran que no existió ningún conflicto de interés en el presente reporte. 